\title{
The role of geometry in the friction generated on the colonic surface by mucoadhesive films
}

\author{
Dimitra Dodou, ${ }^{\text {a) }}$ Paul Breedveld, and Peter A. Wieringa \\ Department of BioMechanical Engineering, Faculty of Mechanical, Maritime and Materials Engineering, \\ Delft University of Technology, Delft 2628 CD, The Netherlands
}

(Received 12 January 2006; accepted 18 April 2006; published online 6 July 2006)

\begin{abstract}
One of the main challenges in designing diagnostic devices able to move along the colon is their locomotion method. Generating friction without applying high normal forces is a key requirement in order to eliminate the risk of tissue damage. One possible solution is to generate friction by means of adhesive forces. For this reason, the device can be covered with mucoadhesive films, which are able to stick on the colonic surface. This paper identifies that the friction of mucoadhesive films depends strongly on their geometry. It seems therefore feasible to adapt the level of the friction to the properties of the colonic surface by altering the geometry of the films covering the device. Moreover, an eventual influence of the film geometry on the generated friction can reveal film shapes which create high grip despite their small size, leading to a decrease in the overall size of the device. The aim of the paper is to test in vitro the relationship between film geometry and generated friction, to fit the experimental findings in a simplified but generic macroscopic theoretical model able to predict the behavior of various geometries, and to embed those findings in the principles of the design implementation. (C) 2006 American Institute of Physics. [DOI: 10.1063/1.2209070]
\end{abstract}

\section{INTRODUCTION}

Colonoscopy is a standard medical procedure in which a long flexible endoscope is inserted into the rectum for inspection of the colon. Pushing the endoscope along the colon can cause painful cramps to the patient and risk perforation of the colonic wall. ${ }^{1,2}$ The drawbacks of the conventional instrumentation have led to research into alternative colonoscopic devices. ${ }^{3-6}$ Such devices should be able to investigate the colonic wall and perform simple interventions (e.g., taking biopsies) like a conventional colonoscope does, eliminating at the same time the risk of pain, damage, or perforation of the colonic wall. For this reason, alternative colonoscopic devices should propagate along the colon preferably by being steered and pulled from the front, instead of being pushed from behind like a conventional flexible endoscope. The main challenge for the development of such devices is therefore their method of locomotion. The devices should be able to generate high static friction with the slippery colonic surface to avoid accidental slip. Moreover, in order for the devices to adjust their locomotion to the varying conditions of the colonic surface, their ability to manipulate the level of the generated friction should be considered.

At the TU Delft, a method to increase static friction with the colonic surface by means of mucoadhesives is being developed. ${ }^{5}$ Mucoadhesives are polymers that can interact with the mucus which covers the colonic surface by forming bonds. Mucoadhesives are originally used as vehicles for controlled and local drug delivery. They can be prepared in a number of semisolid or solid forms, such as hydrogels, films,

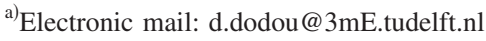

microspheres, sponges, tablets, or microtablets. It has been shown experimentally that mucoadhesives can be used as an interlayer between a colonoscopic device and the colonic surface and that mucoadhesive films, in particular, can generate high static friction, meeting the requirement for safe grip to the colon. ${ }^{6}$ In order to investigate whether friction in the colon can be manipulated using mucoadhesive films, we measured in vitro the static friction generated on porcine intestinal tissue by films of different sizes and reported that friction depends significantly on the area of the films. ${ }^{7}$ It seems therefore feasible to manipulate friction by altering the size of the films which are in contact with the colonic surface (Fig. 1).

Given that the friction generated by mucoadhesive films depends on their size, the shearing of a film on the colonic surface resembles the behavior of adhesive joints. The shear strength of an adhesive joint depends not only on the size, ${ }^{8}$ but also on the geometry of the joint. Giare et al. compared elliptical and rectangular geometries with the same size of overlap and found that shear strength in the first case can be up to eight times higher, because of different shear stress

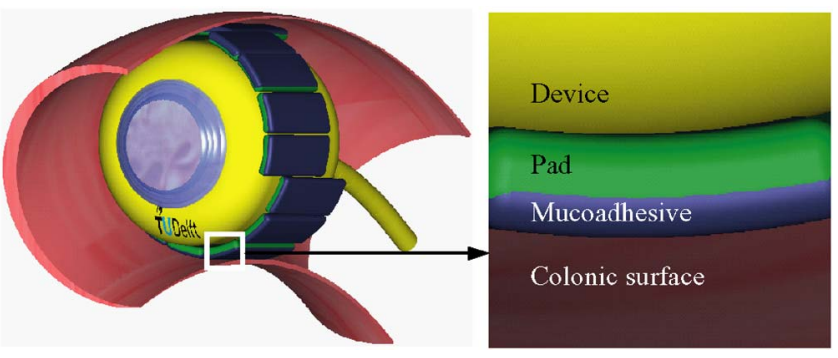

FIG. 1. (Color online) Design concept of an inspection colonoscopic device with mucoadhesive films on the pads and detail of its interface with the colonic surface. 
profile along the adhesive joint. ${ }^{9}$ Considering the resemblance of mucoadhesives with the adhesive joints, the influence of the geometry on the friction of mucoadhesive films with the colonic surface should be further investigated, since it enables friction manipulation by altering the device shape or surface characteristics. Moreover, an eventual influence of the film geometry on the generated friction can reveal film shapes which create high grip despite their small size, leading to a decrease in the overall size of the colonoscopic device. In other words, are the rectangular device pads in Fig. 1 the best shape choice for high static friction? It should be noted, however, that the behavior of a mucoadhesive film on the colonic surface differs from an adhesive joint. In the first case, at least one of the adherands (i.e., the colonic surface) is soft, compliant, and more elastic than the adhesive interlayer (i.e., the mucoadhesive film), whereas the two adherands of an adhesive joint are usually stiff. This difference implies that the principles, theory, and empirical laws which are applicable in the case of adhesive joints can be used only to a limited extent in the case of mucoadhesive films. A modified theory should therefore be established.

The goal of this paper is to verify experimentally whether and to which extent the geometry of mucoadhesive films influences the static friction on the colonic surface. A number of primary geometries were tested in vitro and their impact on the static friction was related to their geometric characteristics. Based on those experimental results, a simplified theoretical model was made able to predict the frictional behavior of films with arbitrary geometries and to indicate eventual design optima of film shapes and sizes.

\section{INITIAL EXPERIMENTS}

The goal of the experiments described below was to investigate whether the geometry of mucoadhesive films influences the friction with the colonic surface. All experiments were carried out in vitro using porcine colons.

\section{A. Materials}

As a mucoadhesive polymer, Carbopol 971P NF (CP971) was used. ${ }^{10}$ Carbopol is the commercial name of high molecular weight cross-linked polymers of acrylic acid, developed by the company Noveon. Carbopols seem to be promising candidates for friction manipulation, since they attach to the mucus via physical bonds (ionic, hydrogen, and van der Waals) that are formed instantaneously. CP971 has a medium degree of cross-linking and molecular weight of 1250 000. ${ }^{11,12}$ Triethanolamine (TEA) was used to neutralize the Carbopol dispersions and polyvinylpyrrolidone (Plasdone ${ }^{\circledR}$ K-90D) (PVP) was used as a film-casting polymer. Propylene glycol was used to strengthen the films and to prevent them from breaking during decasting and storage. Methylene blue was used to stain the film and to assist the observation of their behavior during the experiments.

CP971 was a gift from the company Noveon Inc. (Cleveland, OH, USA) and PVP was a gift from the company ISP Technologies Inc. (Netherlands). TEA and methylene blue were purchased from the company Sigma-Aldrich Chemie BV (Netherlands). All animal procedures were performed using institutionally approved protocols.

\section{B. Preparation of mucoadhesive films}

Mucoadhesive films were prepared according to a method described by Eouani et al. ${ }^{13}$ A $0.3 \%$ w/w mucoadhesive hydrogel was prepared by slowly sifting CP971 into the vortex of distilled water dyed with $0.15 \%$ w/w methylene blue, while stirring at $800 \mathrm{rpm}$. After the entire quantity of dry polymer was introduced, stirring continued for $15 \mathrm{~min}$ at moderate speed $(600 \mathrm{rpm})$ to avoid air entrapment into the dispersion. Then, a small quantity of TEA was added dropwise under mild stirring (500 rpm), until neutralization of the dispersion. ${ }^{11}$ In this way, transparent, lump-free hydrogel dispersions were obtained.

A $10 \% \mathrm{w} / \mathrm{w}$ PVP aqueous solution was prepared under stirring at $800 \mathrm{rpm}$ for $15 \mathrm{~min}$. A volume of $0.6 \% \mathrm{w} / \mathrm{w}$ propylene glycol solution equivalent to the volume of the PVP solution was prepared. The hydrogel dispersion, the PVP solution, and the propylene glycol solution, were mixed under stirring at $800 \mathrm{rpm}$ for $15 \mathrm{~min}$. The produced dispersions were kept overnight at $4{ }^{\circ} \mathrm{C}$ to complete hydration and eventually released entrapped air. The dispersions were then returned to room temperature and poured into Petri dishes. Next, the produced samples were dried in an oven at $38{ }^{\circ} \mathrm{C}$ for $18 \mathrm{~h}$ and the obtained films decasted. The thickness of the films was measured and they were stored at room temperature for at least $48 \mathrm{~h}$ before use. The thickness of the films was in all cases $5 \pm 0.5$ microns.

\section{Methods}

The colon of a pig was extracted, opened longitudinally, and stabilized on a heating pad with the inner surface up to maintain the temperature at $37{ }^{\circ} \mathrm{C}$ (Fig. 2). A mucoadhesive film was fixed to a rectangular Plexiglas plate. The Plexiglas plate was loaded with $100 \mathrm{~g}$ and connected via a thread and a pulley of negligible friction to the force sensor of a tensile testing machine (Zwick 1484). The applied load was selected such that the pressure on the colonic surface was within the range of values of the intra-abdominal pressure ${ }^{14,15}$ on the colon of the human body. The tensile testing machine pulled the Plexiglas plate forward with constant speed and recorded the trace of the generated friction force.

\section{Experiment 1: Effect of geometry}

The goal of experiment 1 was to investigate whether the area of the films is the only parameter which influences the generated static friction. If so, films of varying geometries but with the same area should generate the same amount of friction. Mucoadhesive films were fixed on five plates of different geometry but with the same area (Table I). The edges of the Plexiglas plates were slightly rounded, since sharp edges can cause damage to the colonic wall. For each measurement, a different intestinal segment was used, since the presence of remains of old mucoadhesive films may in- 

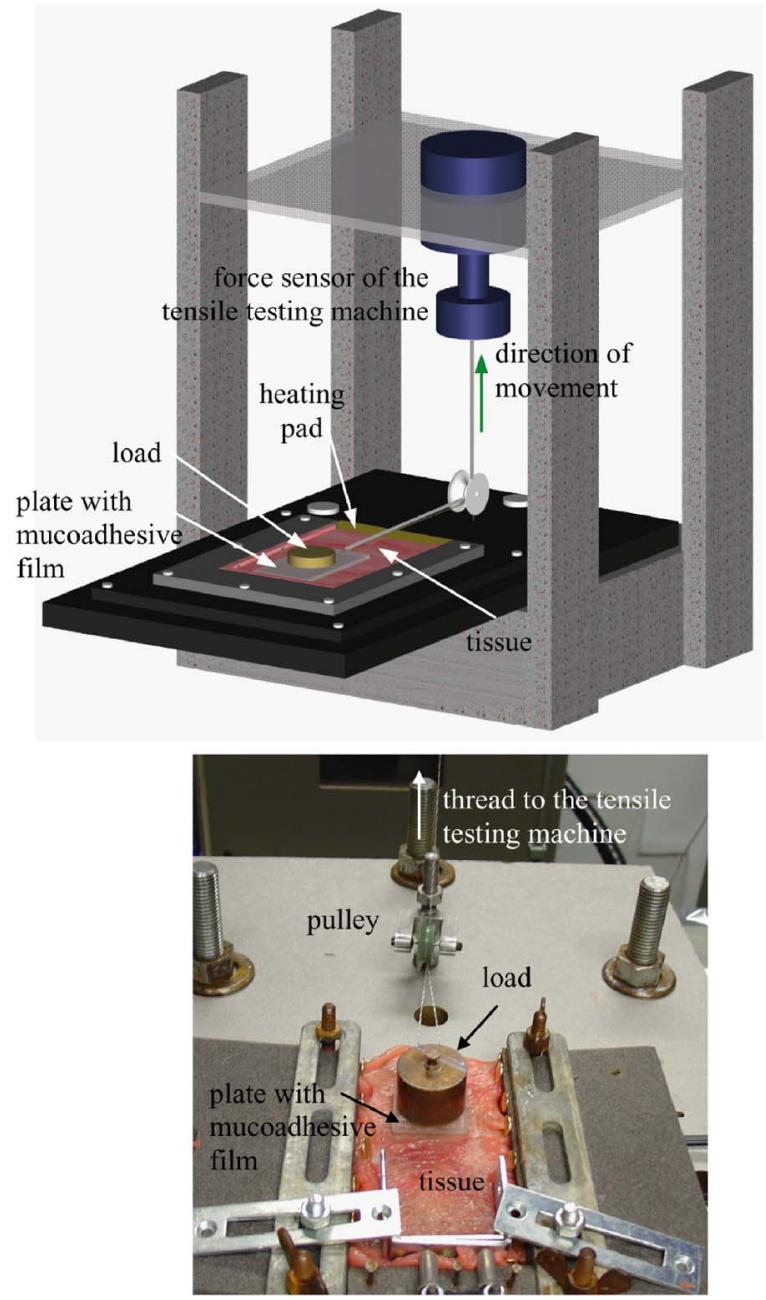

FIG. 2. (Color online) Experimental setup for measuring friction between the inner colonic surface and a mucoadhesive film attached on a plate.

fluence the properties of the mucus. Each plate was tested on two animals, using four tissue segments of each animal. Each plate was thus tested a total of eight times.

\section{E. Experiment 2: Effect of hole position}

The goal of experiment 2 was to investigate whether the static friction generated by mucoadhesive films depends on the position of holes in the film. Mucoadhesive films were fixed on seven square plates of the same size with a single hole placed in different positions (Table II). The edges of the Plexiglas plate were slightly rounded. For each measurement, a different intestinal segment was used. Each plate was again tested on two animals, using four tissue segments of each animal. Each plate was thus tested a total of eight times.

\section{F. Experiment 3: Effect of film orientation}

The goal of experiment 3 was to investigate whether the static friction generated by mucoadhesive films depends on the direction of shearing. Mucoadhesive films were fixed on five rectangular plates with one gradually increasing dimension. The edges of the plate were slightly rounded. For each measurement, a different intestinal segment was used. Each plate was tested in two different directions of shearing (Table III). In this way, two experimental series were created. In series A, the influence of the increasing area and plate length on the friction was investigated, while the plate width was kept constant. In series B, the influence of the increasing area and plate width on the friction was investigated, while the plate length was kept constant. Both series were tested in two animals, using for each plate four tissue segments of each animal. Each plate was thus tested a total of eight times in series $\mathrm{A}$, and eight times in series $\mathrm{B}$.

\section{RESULTS}

\section{A. Experiment 1: Effect of geometry}

The results of experiment 1 [Fig. 3(a)] show that static friction depends significantly on the geometry (Anova $F$ $=262.15, p=0$ ). A triangular geometry generates the lowest friction of all five geometries tested. A square with no holes generates four times lower friction than a square of the same area but with four holes. A circular geometry generates higher friction than a square, but the presence of a hole in this case does not increase friction further.

\section{B. Experiment 2: Effect of hole position}

The results [Fig. 3(b)] show that the static friction generated by mucoadhesive films does not vary significantly with the position of holes in the film (Anova $F=2.27, p$ $=0.05$ ).

TABLE I. Geometric characteristics of the plates tested in experiment 1. Dimensions are in millimeters.

\begin{tabular}{|c|c|c|c|c|c|}
\hline \\
direction \\
of shearing
\end{tabular}


TABLE II. Geometric characteristics of the plates tested in experiment 2. Dimensions are in millimeters.

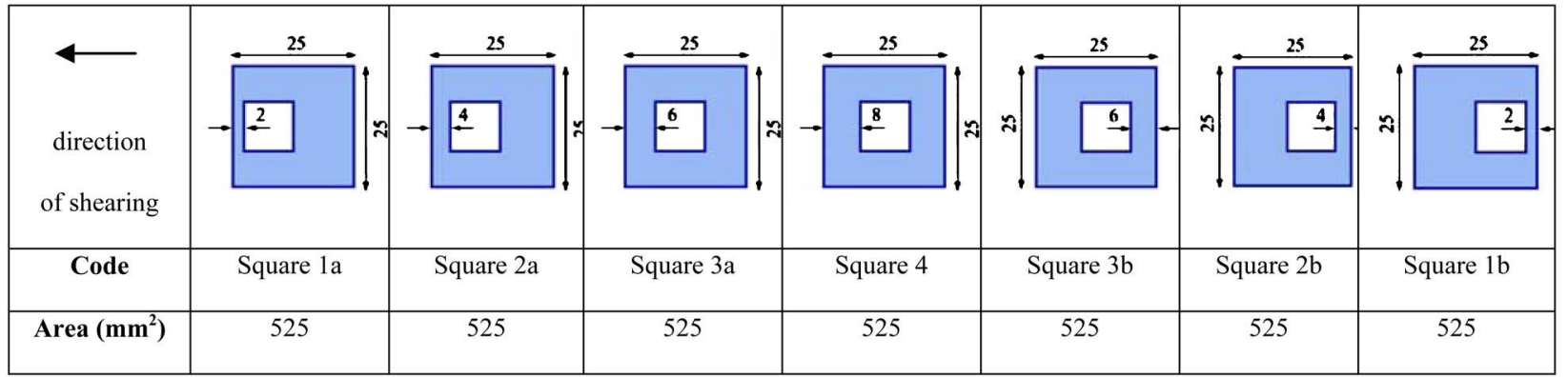

\section{Experiment 3: Effect of film orientation}

As expected and proven earlier, ${ }^{6}$ the generated static friction increases significantly with the area of the mucoadhesive film (Anova $F=6.82, p<0.001$ ) (Fig. 4). To check the influence of the film orientation on the generated friction, a Wilcoxon rank sum test was performed for each of the five plates sheared in two different directions. The results showed that the orientation of the plate during shearing does not influence the generated friction significantly. It should be noted, however, that all plates (except for plate 2 in which two dimensions are almost equal) generated slightly higher, although not significantly different, friction when tested with their longer dimension parallel to the direction of shearing. This can most probably be related to the orientation of irregularities and wrinkles on the colonic pattern, which lie mainly perpendicular to the direction of shearing.

\section{TOWARDS A THEORETICAL MODEL}

\section{A. Friction of mucoadhesives versus shearing of adhesive joints}

In experiments $1-3$, a number of geometries have been tested systematically. The results indicate that the friction between the colonic surface and mucoadhesive films depends strongly on the film geometry. The question now is how we can incorporate the geometry parameter in a generalized friction law and predict the behavior of other geometries as well. Although the theories of adhesive joints incorporate the contribution of the joint geometry on the generated resistant forces, those theories are not applicable in the case of mucoadhesive films. This is mainly due to the fact that in the case of mucoadhesive films, and contrary to the case of adhesive joints, at least one of the adherands (i.e., the colonic surface) is soft, compliant, and more elastic than the adhe-

TABLE III. Geometric characteristics of the geometries tested in experiment 3. Dimensions are in millimeters.

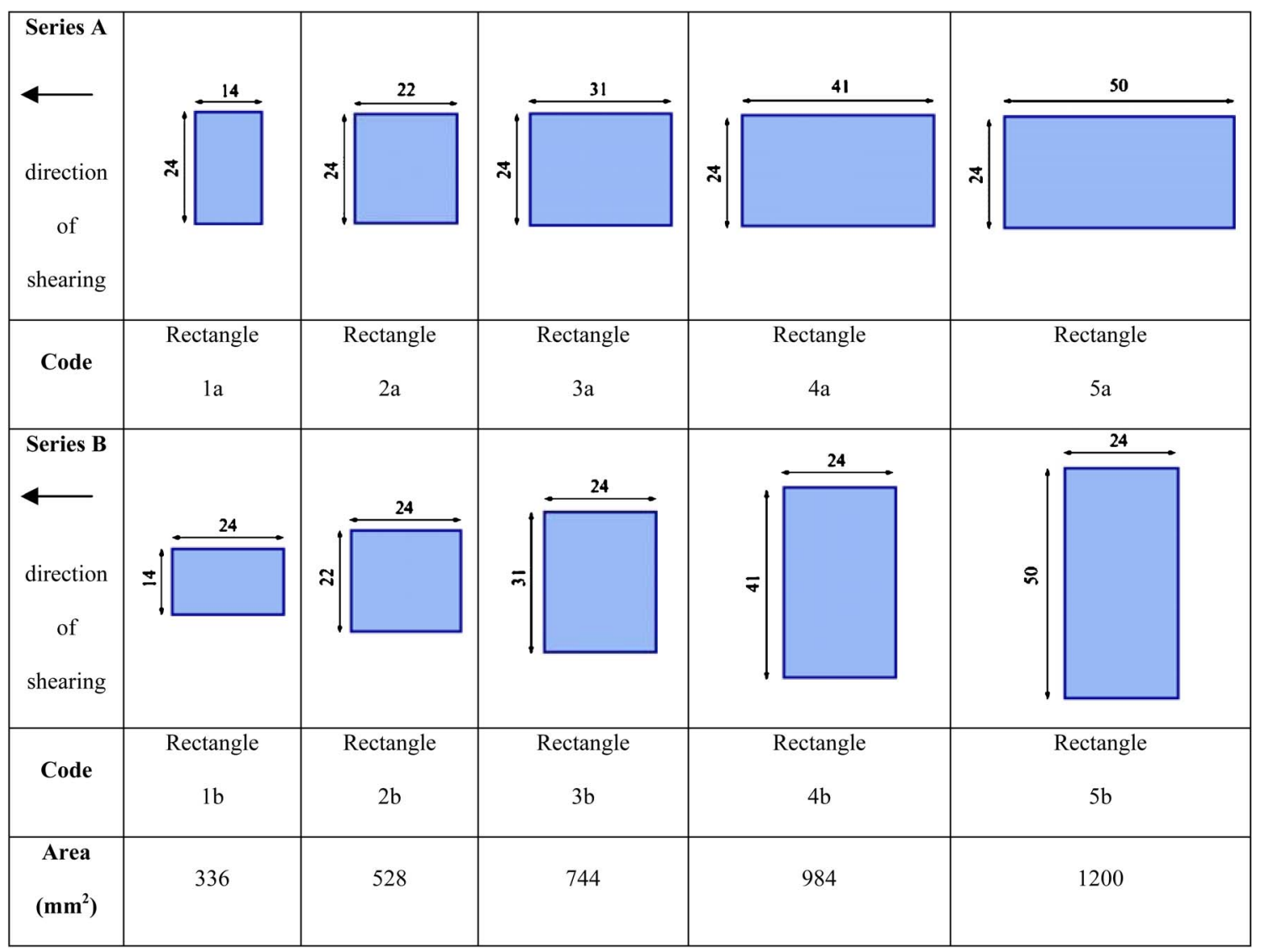




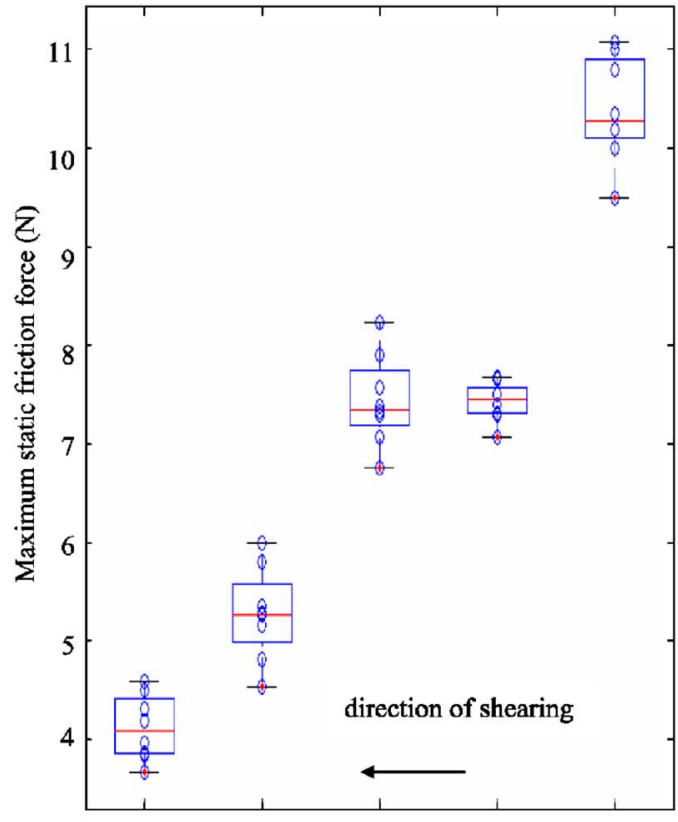

(a)
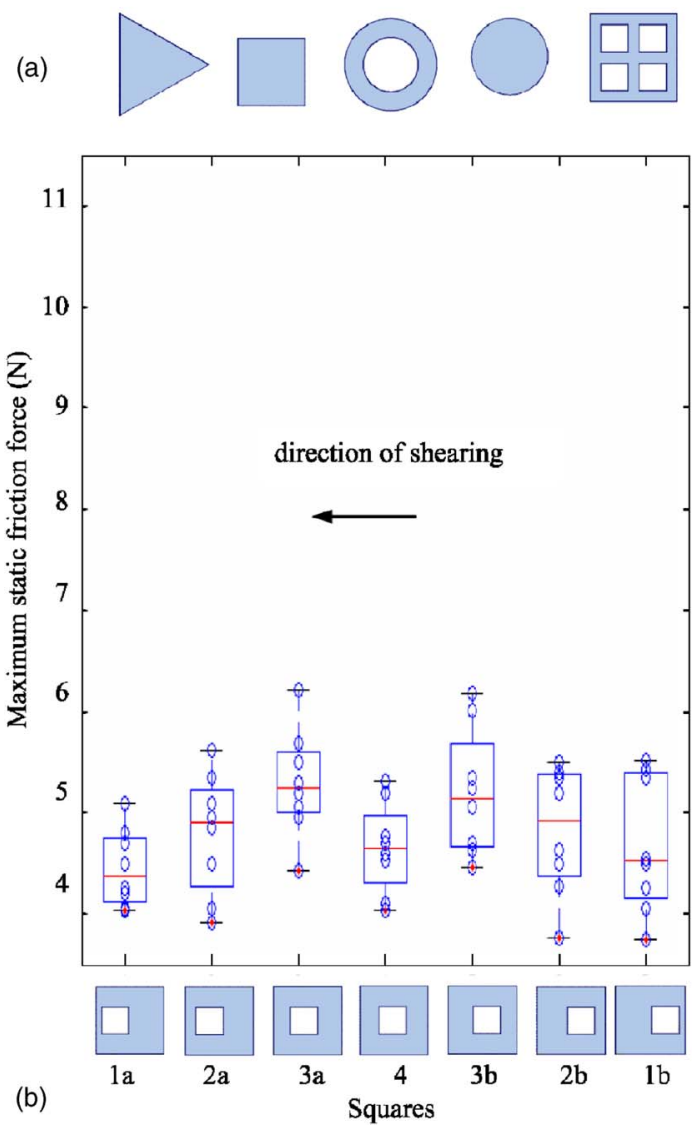

FIG. 3. (Color online) Box plots and experimental data of the results of experiments 1 and 2 showing the maximum static friction force between the colonic surface and mucoadhesive films of (a) varying geometries and (b) varying hole position in the same geometry. The load on the colonic surface was $100 \mathrm{~g}$ in all cases. The line in the middle of the box is the sample median. The lower and upper lines of the box indicate the interquartile range. The whiskers extending out of the box indicate the spread. Noncentered boxes indicate skewness in the results.

sive interlayer (i.e., the mucoadhesive film). In contrast with the significant effect of holes on the friction force of a mucoadhesive structure, researchers have reported, for instance, that the presence of holes along an adhesive joint does not

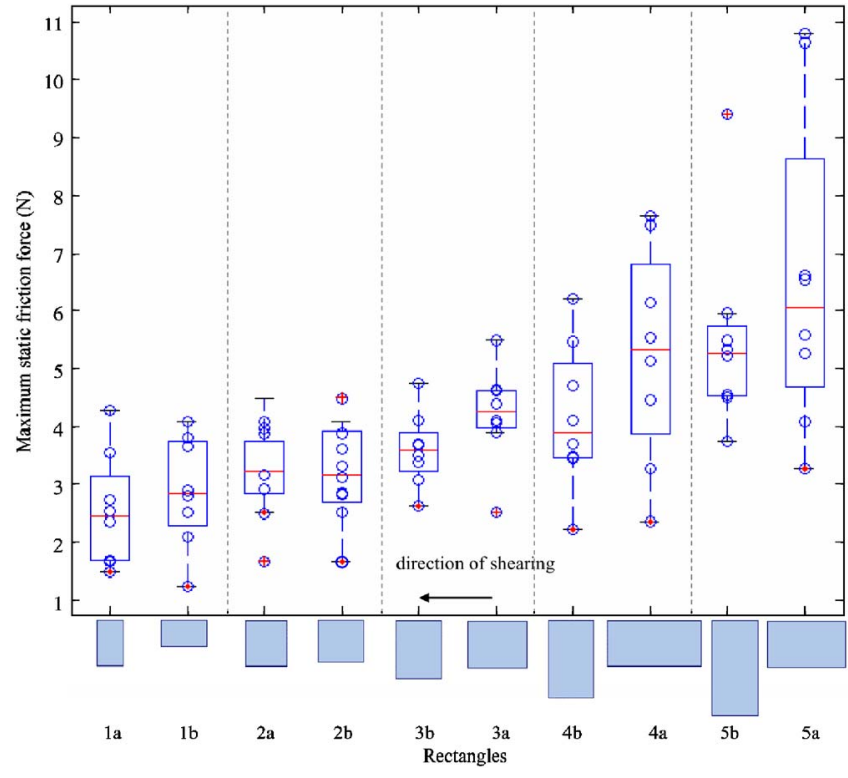

FIG. 4. (Color online) Box plot and experimental data of the results of experiment 3 showing the maximum static friction force between the colonic surface and mucoadhesive films of varying area and direction of shearing. The load on the colonic surface was $100 \mathrm{~g}$ in all cases. The line in the middle of the box is the sample median. The lower and upper lines of the box indicate the interquartile range. The whiskers extending out of the box indicate the spread. Noncentered boxes indicate skewness in the results.

influence the generated friction. ${ }^{8}$ A different approach is therefore required to describe the effect of geometry on the friction with the colon.

\section{B. Friction of hard metals on soft substrates}

In 1943, Bowden et al. developed a theory to make an approximate calculation of the friction of a clean metal block sheared on a softer metal substrate. ${ }^{16}$ When a metal block lies on a softer metal substrate, it sinks into the substrate up to the extent that the contact area between the two metals is able to support the applied load. The theory was formulated in terms of the physical properties of the metals. In brief, Bowden et al. suggested that the friction force $F_{\text {fric }}$ can be written as

$$
F_{\text {fric }}=F_{\text {shear }}+F_{\text {plough }} \text {, }
$$

in which $F_{\text {shear }}$ is the force required to shear the metallic junctions under the metal block and $F_{\text {plough }}$ is the force required to displace the softer metal from the front of the block sideways.

Because of the substrate softness, the real contact area $A_{\text {real }}$ under the metal block can be approximated to the horizontal projected area $A_{\text {proj,horiz }}$ of the metal block on the soft metal substrate (Fig. 5). Similarly, the front area of the sunken metal block can be approximated to the vertical projected area $A_{\text {proj,vert }}$ of the sunken metal block on the soft metal substrate.

The variable $F_{\text {shear }}$ can be expressed as

$$
F_{\text {shear }}=s_{\text {soft metal }} \times A_{\text {proj,horiz }},
$$

in which $s_{\text {soft metal }}$ is the shear strength of the substrate-block interface, i.e., the force per unit area which acts tangentially to the metal interface and is required to shear the adhered 


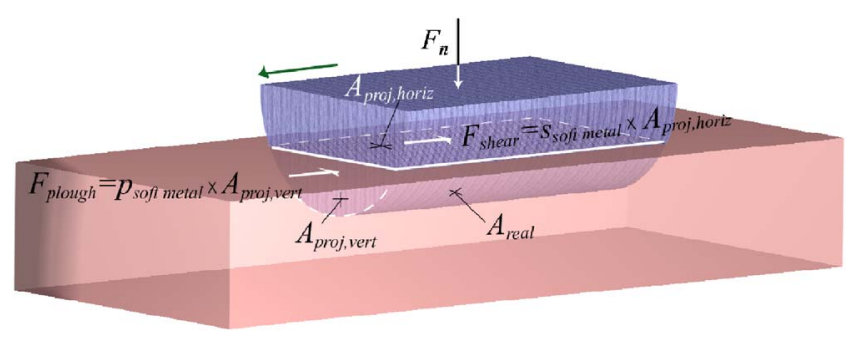

FIG. 5. (Color online) Hard metal block sinking into a soft metal substrate. The green arrow indicates the direction of shearing. $F_{n}$ is the applied load, $F_{\text {shear }}$ is the resistance to shearing, $F_{\text {plough }}$ is the resistance because of sinking, $s_{\text {soft metal }}$ is the shear strength of the substrate-block interface, $p_{\text {soft metal }}$ is the flow pressure of the soft metal, $A_{\text {real }}$ is the real contact area, $A_{\text {proj,horiz }}$ is the horizontal projected area, and $A_{\text {proj,vert }}$ is the vertical projected area.

metal junctions. The variable $F_{\text {plough }}$ can be expressed as

$$
F_{\text {plough }}=p_{\text {soft metal }} \times A_{\text {proj,vert }},
$$

in which the parameter $p_{\text {soft metal }}$ is the flow pressure, i.e., the pressure that causes plastic flow of the softer metal. Plastic flow occurs under loading to increase the contact area under the block up to the extent that it suffices to support the applied load. The flow pressure $p_{\text {soft metal }}$ is therefore a material parameter that correlates the applied load $F_{n}$ with the real contact area $A_{\text {real }}$. Since the real contact area $A_{\text {real }}$ is approximated to $A_{\text {proj,horiz, }}$, we can write that

$$
p_{\text {soft metal }}=\frac{F_{n}}{A_{\text {proj,horiz }}} .
$$

As Bowden et al. already commented, ${ }^{16}$ the term of flow pressure is not a clearly defined property of a particular metal. To express eventual differences to the sinkage degree derived by the particular properties of the soft substrate, we could introduce in Eq. (4) a material parameter $H$ of the soft substrate, i.e.,

$$
p_{\text {soft metal }}=\frac{F_{n}}{A_{\text {proj, horiz }}} H .
$$

\section{Friction of hard metals on soft substrates supported by an underlying hard metal surface}

Bowden et al. explained that the term $F_{\text {plough }}$ is usually small so that the deviations from Coulomb's law are not noticeable. They further investigated experimentally the role of the applied load and its correlation with the contact area, particularly in the case that a metal block lies on a softer metal substrate which again is supported by an underlying hard metal surface. As referred above, when a metal block lies on a softer metal substrate, it sinks into the substrate up to the extent that the contact area between the two metals is able to support the applied load. When the soft metal substrate is supported by an underlying hard metal surface, however, increasing the applied load after an upper threshold will not lead to further sinking into the soft metal substrate or further increase of the contact area, since the underlying hard metal surface prevents stronger deformation of the soft metal substrate. As a result, the generated friction becomes almost independent of the load when the applied load $F_{n}$ exceeds an

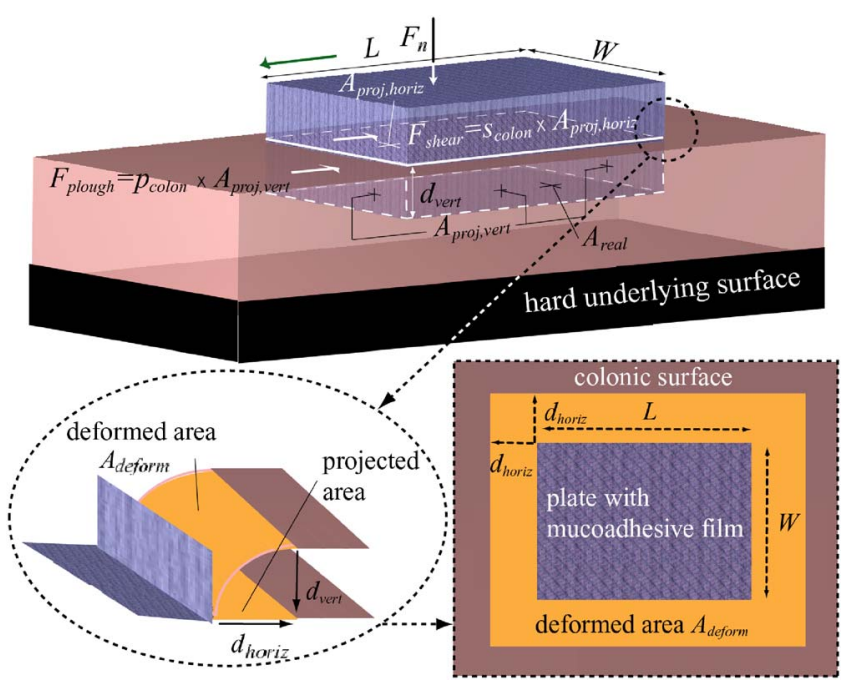

FIG. 6. (Color online) Plate with mucoadhesive film sinking into soft colonic tissue. The green arrow indicates the direction of shearing. $F_{n}$ is the applied load, $F_{\text {shear }}$ is the resistance to shearing, $F_{\text {plough }}$ is the resistance because of sinking, $s_{\text {colon }}$ is the shear strength of the colon-mucoadhesive interface, $p_{\text {colon }}$ is the flow pressure of the colonic tissue, $A_{\text {real }}$ is the real contact area, $A_{\text {proj,horiz }}$ is the horizontal projected area, $A_{\text {proj,vert }}$ is the vertical projected area, $d_{\text {vert }}$ is the deformation depth, $W$ is the width of rectangular plate, and $L$ is the length of rectangular plate.

upper threshold. In other words, the load influences the generated friction only to the extent that it influences the contact area. $^{16}$

\section{Mucoadhesive films on the colonic surface versus hard metals on soft substrates}

A plate with a mucoadhesive film lying on a soft colonic segment that is supported by an underlying hard surface presents similarities with the cases investigated by Bowden et al. Their approach was therefore revisited and used as the starting point for developing a theory that is able to predict the friction of the mucoadhesive films in terms of their physical and geometric characteristics. It should be noted, however, that a number of differences between the two cases exist, such as the role of the film: whereas the eventual presence of a film between the two metals is usually contaminant and deteriorates the intimacy of the contact and the strength of metal adhesion, ${ }^{16}$ the presence of a mucoadhesive film is the key factor which provides intimacy of contact and adhesive strength within the plate-colon interface. Additionally, we reconsidered the term of flow pressure and adjusted it to the case in which the soft metal substrate is replaced by the highly anisotropic colonic tissue.

\section{E. Rectangular plate with mucoadhesive film lying on the colonic surface}

Description of the geometry. Consider a rectangular plate of width $W$ and length $L$. The plate is covered by a mucoadhesive film, is loaded with a load $F_{n}$, and lies on a segment of colonic tissue that is supported by an underlying hard surface (Fig. 6). The friction force $F_{\text {fric }}$ between the film and the tissue can then be expressed as 


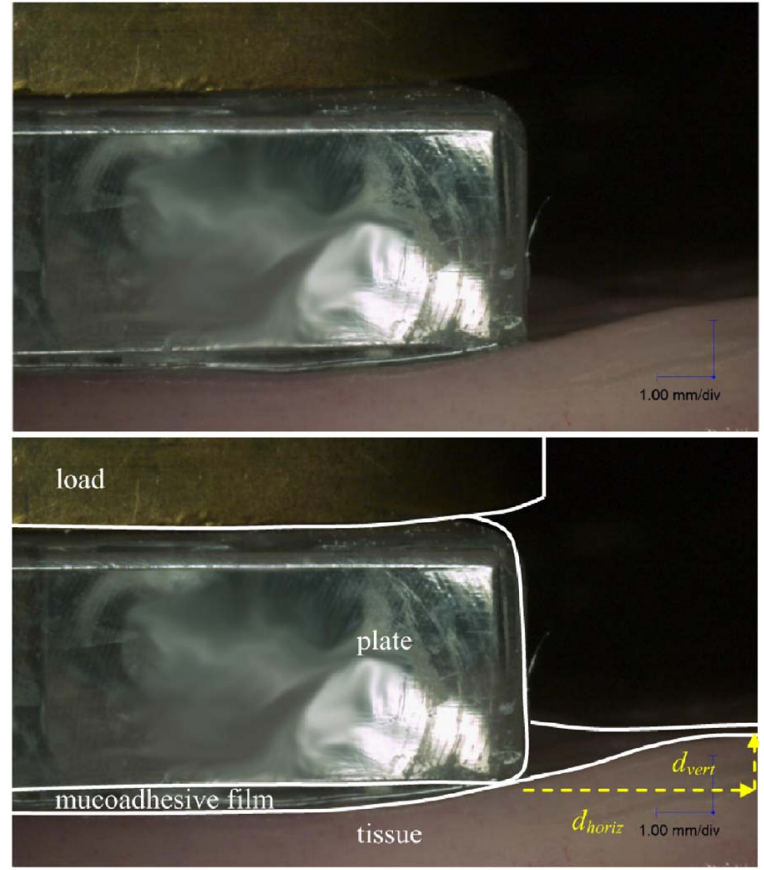

FIG. 7. (Color online) Microscopic front picture of a plate covered with a mucoadhesive film lying on the tissue and loaded with load $F_{n}$ (top). In the photo at the bottom, white lines are added to emphasize the contours. $d_{\text {horiz }}$ is the horizontal distance up to which the tissue is deformed and $d_{\text {vert }}$ is the deformation depth.

$$
F_{\text {fric }}=F_{\text {shear }}+F_{\text {plough }}
$$

in which $F_{\text {shear }}$ is the resistance to shearing and $F_{\text {plough }}$ the resistance because of sinking.

Calculation of $F_{\text {shear. }}$. For the case of a mucoadhesive in contact with the mucus layer, visualization studies of the interface showed that no serious interpenetration of the free chains occurs in a microscopic range. ${ }^{17}$ The interface appeared as an irregular but sharp borderline. We can therefore approximate the real contact area $A_{\text {real }}$ under the film to the horizontal projected area

$$
A_{\text {proj,horiz }}=W \times L \text {. }
$$

The variable $F_{\text {shear }}$ can hence be calculated as

$$
F_{\text {shear }}=s_{\text {colon }} \times A_{\text {proj,horiz }}=s_{\text {colon }} \times W \times L,
$$

in which $s_{\text {colon }}$ is the shear strength of the colonmucoadhesive interface.

Calculation of $F_{\text {plough }}$. Because the colonic tissue is soft, the plate with the film sinks into the tissue up to a deformation depth $d_{\text {vert }}$ (Figs. 6 and 7). It can be further assumed that, because of the presence of a mucoadhesive film and in contrast with the cases investigated by Bowden et al., not only the front but also the back and side sunken areas of the sheared plate contribute to the generated friction. Whereas the front faces an accumulation of tissue which acts like a mechanical obstruction against motion, the back resists to peeling and the sides to shearing of the mucoadhesive film. It should thus be considered that the front and back areas contribute to the friction with a different weight factor than the side areas, since the underlying phenomena differs. Hence,

$$
F_{\text {plough }}=p_{\text {colon }} \times A_{\text {proj,vert }},
$$

in which $A_{\text {proj,vert }}$ is a weighted function of the front, back, and side areas (Fig. 6) and $p_{\text {colon }}$ is the flow pressure of the colonic tissue. In the case of the rectangular plate, $A_{\text {proj,vert }}$ can be calculated as

$$
A_{\text {proj, vert }}=2\left(f_{\text {weight } W} W+f_{\text {weight } L} L\right) d_{\text {vert }},
$$

in which $f_{\text {weight } W}$ and $f_{\text {weight } L}$ are correction factors differentiating the contribution of the back and front areas from that of the side areas. It should be noted that the same correction factor $f_{\text {weight } W}$ was used to express the contribution of both the back and the front sunken areas to the friction, although two completely different phenomena (film detachment and mechanical obstruction, respectively) occur. The reason was that in both cases the resulting forces are perpendicular to the applied areas (or planes if one prefers), whereas the force generated by the side sunken areas acts parallel to the applied plane. In a more elaborate approach, however, the influence of the back and front can be further separated.

Reconsidering the flow pressure. The presence of the highly deformable and anisotropic colonic tissue generates the need to reconsider the flow pressure as defined for the case of soft metal substrates. When a load $F_{n}$ is applied on the plate and the tissue is considerably flexible, the tissue deformation will not be limited to the area under the plate, but will be extended around the plate as well. In other words, the area which supports the applied load is larger than the projected area of the plate $A_{\text {proj,horiz, }}$, so that an area correction should be introduced when determining the term of the flow pressure.

Since the flow pressure depends on the material properties of the soft tissue, in particular, on the ability of the soft tissue to subside under loading, we included the dimensionless hardness $H_{\text {colon }}$ of the soft tissue when defining the flow pressure. Hence,

$$
p_{\text {colon }}=\frac{F_{n}}{\left(A_{\text {proj,horiz }}+A_{\text {deform }}\right)} H_{\text {colon }},
$$

in which $A_{\text {deform }}$ is the area of the tissue around the plate which deforms under loading because of tissue flexibility (area correction). The hardness of the soft tissue $H_{\text {colon }}$ is taken from the literature. Since no information about the hardness of colonic tissue was found in the literature, we used initially the hardness of skin, ${ }^{18}$ considering that skin is a soft and thin tissue similar to the colon. Further research is required to identify the value of $H_{\text {colon }}$ for the colonic tissue more precisely. If we simplify the soft tissue as a network of vertical springs connected together by means of horizontal springs, then the hardness $H_{\text {colon }}$ would describe the stiffness and density of the vertical springs, whereas the term $A_{\text {deform }}$ would be due to the presence of horizontal springs which transfer the tissue deformation to the neighboring tissue around the plate.

In order to determine $A_{\text {deform, }}$, we approximate the deformed tissue area around the plate to its horizontal projection on the nondeformed tissue. In this way, the deformed area around the plate is approximated to a frame of width $W+2 d_{\text {horiz }}$, length $L+2 d_{\text {horiz }}$ (Fig. 6) and area 


$$
A_{\text {deform }}=\left(W+2 d_{\text {horiz }}\right)\left(L+2 d_{\text {horiz }}\right)-W L,
$$

in which $d_{\text {horiz }}$ is the horizontal distance up to which the tissue is deformed (Fig. 7). The distance $d_{\text {horiz }}$ can be expressed as

$$
d_{\text {horiz }}=\frac{F_{n}}{k_{\text {colon }} \times A_{\text {proj, horiz }}}=\frac{F_{n}}{k_{\text {colon }} \times W \times L},
$$

in which $F_{n} / A_{\text {proj,horiz }}$ is the pressure applied on the colonic tissue at the edge of the plate and $k_{\text {colon }}$ a material parameter connected to the tissue deformability in horizontal direction. Combining Eqs. (6) and (10)-(12), $p_{\text {colon }}$ becomes

$$
p_{\text {colon }}=\frac{F_{n}}{\left[W+2\left(F_{n} / k_{\text {colon }} W L\right)\right]\left[L+2\left(F_{n} / k_{\text {colon }} W L\right)\right]} H_{\text {colon }} \text {. }
$$

\section{F. Calculation of friction}

As defined above, the friction force $F_{\text {fric }}$ between the film and the tissue can then be expressed as $F_{\text {fric }}=F_{\text {shear }}$ $+F_{\text {plough }}=s A_{\text {proj,horiz }}+p_{\text {colon }} A_{\text {proj,vert }}$. Combining Eqs. (6), (9), and (10), the friction force can be calculated by means of the plate and film geometric characteristics and the material properties of the tissue.

$F_{\text {fric }}=s_{\text {colon }} W L$

$$
\begin{aligned}
& +\frac{F_{n}}{\left\{W L+2(W+L)\left(F_{n} / k_{\text {colon }} W L\right)+4\left[\left(F_{n} / k_{\text {colon }} W L\right)\right]^{2}\right\}} \\
& \times H_{\text {colon }}\left[2\left(f_{\text {weight } W} W+f_{\text {weight } L} L\right) d_{\text {depth }}\right] .
\end{aligned}
$$

Similar relationships can be derived for rectangular geometries with holes, circular geometries, and triangular geometries (see the Appendix). When holes were present within a geometry, we assumed that the holes were large enough to keep the deformation depth inside the hole the same as the deformation depth around the plate. For circular geometries, the friction force was split into two components, one parallel and one perpendicular to the tangent at each point of the circumference.

\section{EXPERIMENTAL FITTING}

In order to check the validity of the theory presented above, we applied it for the geometries of Tables I-III. We assumed that the underlying hard substrate is hard enough and the applied load sufficient to submerge all the plates into the tissue up to the same finite deformation depth $d_{\text {vert }}$. The geometric characteristics of the geometries can be measured, whereas the hardness of the soft tissue $H_{\text {colon }}$ is taken from the literature. Since three parameters are unknown $\left(d_{\text {vert }}\right.$, $s_{\text {colon }}$, and $k_{\text {colon }}$ ), three of the geometries (rectangles $3 \mathrm{a}$ and $4 \mathrm{~b}$, and square $2 \mathrm{a}$ in Tables II and III) were used as reference. The mean of their static friction values measured during the experiments was used to determine the values of $d_{\text {vert }}$, $s_{\text {colon }}$, and $k_{\text {colon }}$ using Eqs. (14) and (A10). Then, the friction force was calculated for the rest 19 geometries (Tables I-III) by applying Eq. (14), as well as (A10), (A15), (A21), and (A26). The calculated forces were plotted against the forces

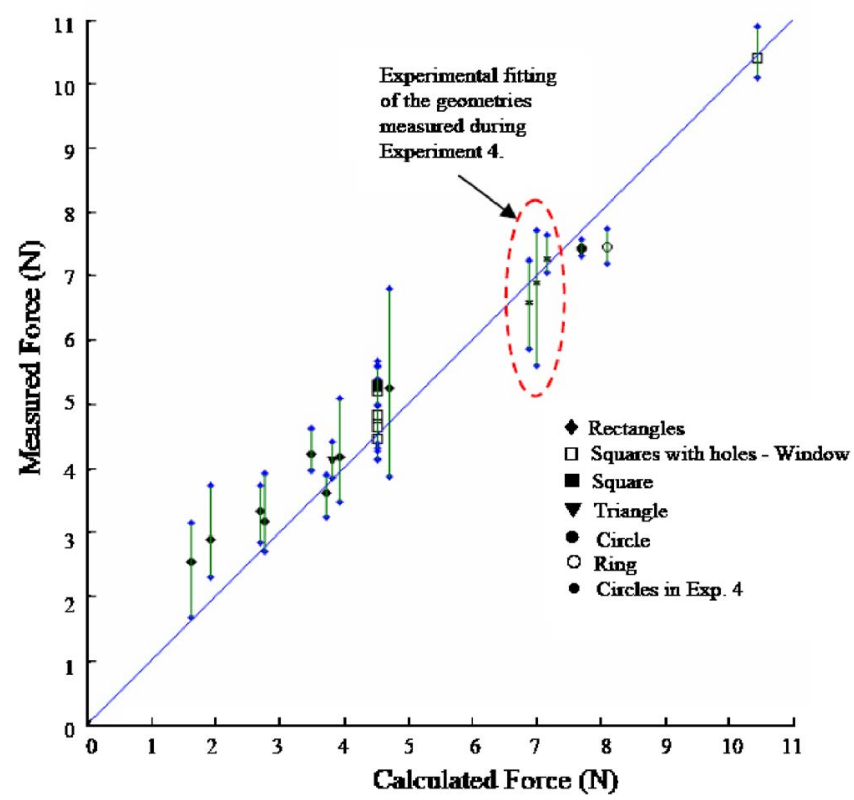

FIG. 8. (Color online) Experimental fitting of experimental static friction values measured for all plates appeared in Tables I-IV with the calculated friction values from Eqs. (14), (A11), (A13), (A15), and (A17). The symbol in the middle is the mean of the measured friction, whereas the green line indicates the interquartile range. The blue line defines the $45^{\circ}$ slope.

measured during the experiments (Fig. 8). The figure shows that the measured friction values fit accurately the calculated friction values, following a slope of $45^{\circ}$.

The three geometries used as reference have been chosen according to the following criteria:

The proposed theoretical model does not introduce the curvature of the borderline as a parameter of influence and all components of the generated force are parallel or perpendicular to the applied areas (or planes). Since rectangles contain exclusively planes that are either parallel or perpendicular to the direction of shearing, only rectangles are used as a reference so that circles and triangles can be used to check the predictive ability of the model regarding shapes (with curvatures or planes that form an angle with the direction of shearing) that are not included in the reference.

(ii) Considering that three kinds of rectangles have been experimentally tested (rectangles with no holes, one hole, or four holes), one plate of each kind was selected as reference.

(iii) Small rectangles were excluded from the reference in order to avoid introducing eventual scale effects in the experimental fitting.

It should be noted that for the experimental fitting of the theoretical model, $H_{\text {colon }}=30$ in shore A hardness scale, value taken from literature source on skin hardness. (Shore A hardness scale is suitable for rubber, soft plastics, and soft biological tissues and can be determined with an instrument called shore A durometer. If the indenter completely penetrates the sample, a reading of 0 is obtained, and if no penetration occurs, a reading of 100 results. The reading is dimensionless.) 
TABLE IV. Geometric characteristics, predicted and measured static friction of the geometries tested in control experiment. The arrow indicates the direction of shearing. Dimensions are in millimeters.

\begin{tabular}{|c|c|c|c|}
\hline & \\
\hline Code & Circle 1 & Circle 2 & Circle 3 \\
\hline Area (mm $\left.\mathbf{m}^{2}\right)$ & 502 & 568 & 602 \\
\hline Predicted friction (N) & 7.16 & 6.99 & 6.88 \\
\hline Measured friction (N) & (interquartile range=0.58) & (interquartile range=1.7) & (interquartile range=1.4) \\
\hline
\end{tabular}

Since the skin and the colon hardness can differ, a sensitivity analysis for the experimental fitting of the theoretical model has been carried out. For this reason, $H_{\text {colon }}$ has been varied between 25 and 50 and its influence on the friction calculated by the model as well as on the values of the three unknown parameters $\left(d_{\text {vert }}, s_{\text {colon }}\right.$, and $\left.k_{\text {colon }}\right)$ has been investigated. We assume that the colon hardness falls into the hardness range between 25 and 50, considering that measured hardness values for silicone rubber, which is often used to simulate a variety of soft tissue, range between 24.6 and 50.2. ${ }^{19}$

It appears that neither the friction values calculated by the theoretical model nor the values of $s_{\text {colon }}, k_{\text {colon }}$ are affected by the changes in $H_{\text {colon }}$. As can be seen from

$$
\begin{aligned}
F_{\text {plough }}= & p_{\text {colon }} A_{\text {proj, vert }} \\
= & \frac{F_{n}}{\left[W+2\left(F_{n} / k_{\text {colon }} W L\right)\right]\left[L+2\left(F_{n} / k_{\text {colon }} W L\right)\right]} \\
& \times H_{\text {colon }} 2\left(f_{\text {weight } W} W+f_{\text {weight } L} L\right) d_{\text {vert }}
\end{aligned}
$$

changes in $H_{\text {colon }}$ are compensated by changes in $d_{\text {vert }}$. More specifically, when $H_{\text {colon }}$ increases from 25 to $50, d_{\text {vert }}$ decreases from 0.636 to $0.318 \mathrm{~mm}$ and therefore within the order of magnitude of $d_{\text {vert }}$ observed microscopically (Fig. 7). Further research will be focused on defining experimentally $d_{\text {vert }}$, so that this parameter will be decoupled from the tissue hardness.

\section{IN QUEST OF THE OPTIMAL SHAPE: CONTROL EXPERIMENT}

To check the predictive ability of the theory for geometries different from those tested during experiments $1-3$, the friction of three geometries, in Table IV, was first calculated and then measured experimentally. Since our main goal is to maximize static friction with the colonic surface and since circular geometries and geometries with holes turned out to lead to high friction, the three geometries have the form of circular structures with holes, in order to investigate whether such a combination can lead to high friction. The experimen- tal method was the same as in experiments 1-3. All plates were tested on the same animal. Each plate was tested eight times.

As shown in Table IV as well as in Fig. 8, the friction generated by circles $1-3$ can be well predicted, indicating that such a theoretical approach can be applicable to a large variety of different geometries. The results of the control experiment show that a geometric configuration with concentric holes can be favorable in comparison with geometric configurations with eccentric holes, generating higher friction despite its smaller area.

\section{DISCUSSION}

\section{A. Friction depends on geometry}

Geometry is of importance when a mucoadhesive film grips on the colonic surface. By means of the theoretical model described above, the level of the generated static friction can be estimated as a function of the film geometric characteristics and the tissue material properties. The model is applicable not only for the mucoadhesive-colonic interface. Estimating optimal shapes for gripping to vulnerable or highly deformable tissues (e.g., grasping in minimally invasive surgery or in haptics) can be achieved if we know the material properties of the soft substrate. Footwear or wheels profile for gripping and moving while sinking into soft snow could be identified as well. It should be noted, however, that the model is considerably simplified and refinement is one of our future goals. It can be already seen, for instance, in Fig. 8 , that small plates at the left of the curve deviate from the $45^{\circ}$ slope and perform better than predicted from the theory. There is apparently a scale effect involved in this inclination. The scale effect is probably connected with the tissue relief (i.e., the presence of irregularities and wrinkles in various scales) and should be further quantified.

\section{B. Transition from static to dynamic friction}

In order to gain a better understanding of the mechanisms involved in the generation of high friction on the colonic surface, the mode of failure of the mucoadhesive films 


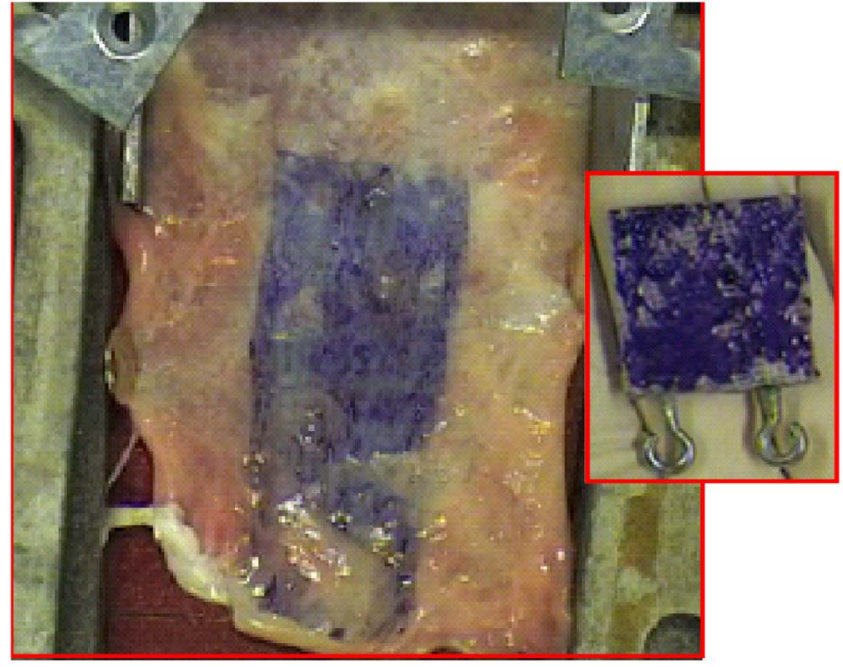

FIG. 9. (Color online) Colonic surface (left) and bottom side of a plate (right) after the plate has been sheared along the colonic surface. The blue footprints are fragments of the mucoadhesive film. Since fragments are present both on the colonic surface and the plate, motion of the plate appears to be initiated mainly by means of cohesive failure of the mucoadhesive film.

is discussed. In general, failure of a mucus-mucoadhesive bond occurs at the weakest component of the joint. According to Hägerstrom and Edsman, ${ }^{20}$ mucoadhesive performance can be assessed by localizing the failure of the mucoadhesive joint. It seems that weaker mucoadhesives fail at their interface with mucus, whereas for stronger mucusmucoadhesive bonding it is initially the mucus and later the hydrated mucoadhesive that fail internally. ${ }^{21}$ In our experiments, no mucus internal fracture was detected and motion was initiated mainly by the cohesive failure of the mucoadhesive and partial adhesive failure at the mucusmucoadhesive interface (Fig. 9).

It should be noted that cohesive failure of an adhesive bond has been supported by a number of independent studies. Researchers found out that cohesive failure required much more work than adhesive failure, producing therefore stronger bonding. ${ }^{22}$ One reaches the paradoxical conclusion that weakening the adhesive bonding can lead in some cases to a stronger joint. In fact, the adhesive bonding must be slightly weaker than the cohesive strength in order to gain the increased dissipation associated with cohesive failure. This can be achieved by cross-linking or entanglement of the adhesive polymer. Moreover, although leaving fragments behind lying on the colonic surface, a mucoadhesive film failing internally offers a method for friction maximization (or control) that remains rather uninfluenced by the properties of the mucus and therefore consistent (at least up to the extent that the properties of the mucus provide sufficient adhesion so that the mucus-mucoadhesive interface does not become the weakest link).

\section{Design implementation}

As a first design approach (Fig. 10), the device can consist of a cylinder with invariable diameter (yellow) covered

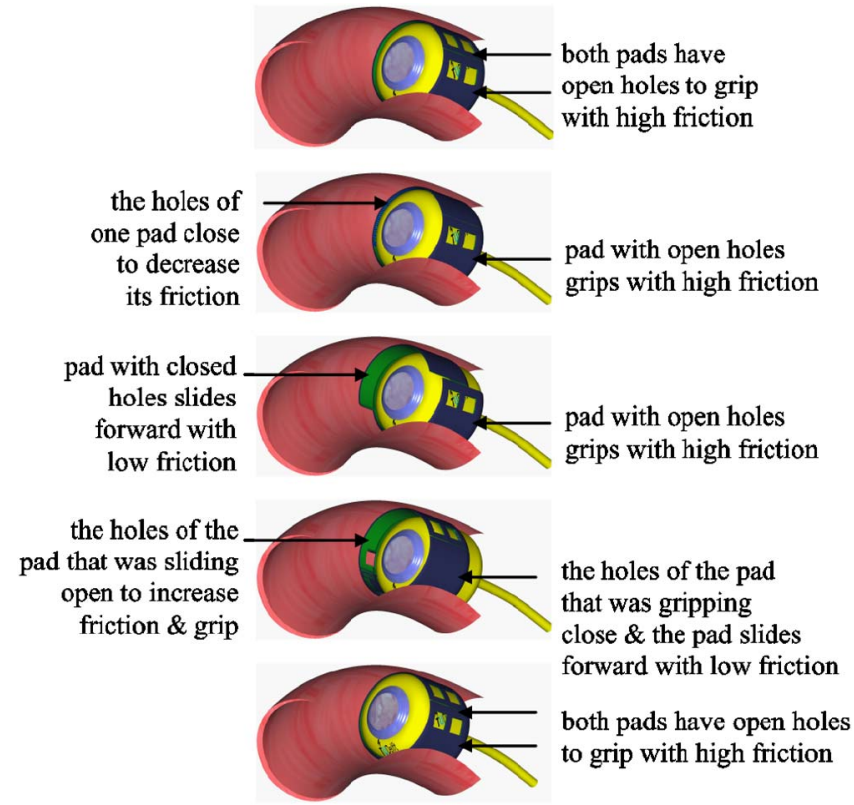

FIG. 10. (Color online) Design concept of a colonoscopic device which manipulates friction by altering the geometry of two pads covered with mucoadhesive films.

with two pads (green) which are coated with mucoadhesive films (blue). The influence of the film geometry on the friction can be used to indicate the shape of the device pads which generates high friction without leading to an increase of the device size. From a design point of view, rectangular pads are more preferable than circular pads. Moreover, it will be desirable to introduce holes in the pads so that the generated friction increases because of the long borderline. Since holes compensate for size, the device can generate high static friction by meeting at the same time the requirement of compactness. The number of holes as well as their distribution (eccentric or concentric) is a question of future design optimization.

The role of holes introduced in the device pads can be further expanded if the holes are used not only for friction increase but also for friction manipulation. This can be achieved if it is possible to open and close the holes on demand. According to the findings presented in this article, a pad with open holes should generate higher static friction than a pad with close holes, such as the squares with holes in Table II which generate higher friction than the square without holes in Table I.

When the device needs to stand on place, both pads have open holes to grip with high static friction. To make one step forward, the holes of one pad close and initiate sliding of the pad with lower friction. As soon as the pad has slid forward, the holes are open so that the pad stops and grips to the colon with high friction. The other pad remains standing with open holes up to the moment that the slid pad is locked in the new position. Then, the pad that was gripping behind closes the holes, slides forward, and stops by opening again the holes. As soon as both pads have slid forward and gripped to the new position, the cylinder that supports the pads slides forward as well, so that the device makes one step further. 


\section{CONCLUSIONS}

The static friction of a mucoadhesive film on the colonic surface depends strongly on the film geometry. By altering the film geometry, we can manipulate the level of the generated friction, and switch between high and low friction values, according to the demands of the colonoscopic device. Moreover, choosing geometries which can achieve high friction despite their compact size can lead to a decrease of the overall size of the colonoscopic device. By developing a generic macroscopic theoretical model, we are able to express the generated friction as a function of the film geometry and the tissue material properties. The generic character of such a simplified approach consists of the fact that in all cases the friction force can be calculated as the sum of two components, one component connected with resistance against shearing and a second component related to the presence of a soft material (the colon) lying on a hard underlying substrate. The future aim is to design a colonoscopic device of small size and versatile shape with the ability to manipulate and adapt the level of the required friction according to the demands of the varying and often harsh conditions of the colonic surface. Testing the device in vivo will indicate the potential and limitations of colonic friction increase and manipulation. Although the absolute values of friction cannot be identical to those derived from in vitro testing, it can be expected that the geometry will continue influencing the generated friction. The influence can be even more expressed than during the in vitro testing, if one considers that the role of the geometry is related to the sinkage into the tissue and sinkage in vivo can be in turn deeper because of the absence of a hard underlying surface.

\section{ACKNOWLEDGMENTS}

This research has been made possible by the Van der Leeuw professorship (Dutch Technology Foundation STW) awarded to one of the authors (P.A.W.). The research of another author (P.B.) has been made possible by a fellowship of the Royal Netherlands Academy of Arts and Sciences.

\section{APPENDIX: DERIVATION OF EQUATIONS FOR FRICTION OF VARIOUS GEOMETRIES}

\section{Rectangular geometries with one or more holes of the same size}

Consider a rectangular plate of width $W$ and length $L$. The plate includes $n$ holes of width $W_{h}$ and length $L_{h}$. We assume that the holes are large enough so that the deformation depth inside a hole is the same as the deformation depth around the plate. Similar to the case of rectangular plates without holes, the friction force $F_{\text {fric }}$ can be calculated as

$F_{\text {fric }}=F_{\text {shear }}+F_{\text {plough }}$,

in which

$$
F_{\text {shear }}=s_{\text {colon }} A_{\text {proj,horiz }}
$$

and

$$
F_{\text {plough }}=p_{\text {colon }} A_{\text {proj, vert }} .
$$

The horizontal projected area can be calculated as

$$
A_{\text {proj,horiz }}=W L-n W_{h} L_{h},
$$

whereas the vertical projected area as

$$
\begin{aligned}
A_{\text {proj, vert }}= & 2\left[\left(f_{\text {weight } W} W+f_{\text {weight } L} L\right)\right. \\
& \left.+n\left(f_{\text {weight } W} W_{h}+f_{\text {weight } L} L_{h}\right)\right] d_{\text {vert }} .
\end{aligned}
$$

The flow pressure is defined as previously, i.e.,

$$
p_{\text {colon }}=\frac{F_{n}}{\left(A_{\text {proj,horiz }}+A_{\text {deform }}\right)} H_{\text {colon }} \text {, }
$$

in which $A_{\text {deform }}$ includes in this case the area correction around the plate $A_{\text {deform, } o}$ as well as the area correction inside the holes $A_{\text {deform, } i}$. Both area corrections can be easily calculated by means of the plate geometric characteristics, i.e.,

$$
A_{\text {deform }, o}=\left(W+2 d_{\text {horiz }}\right)\left(L+2 d_{\text {horiz }}\right)-W L
$$

and

$$
A_{\text {deform, } i}=n W_{h} L_{h}-n\left(W_{h}-2 d_{\text {horiz }}\right)\left(L_{h}-2 d_{\text {horiz }}\right) .
$$

The horizontal distance $d_{\text {horiz }}$ is determined as,

$$
d_{\text {horiz }}=\frac{F_{n}}{k_{\text {colon }} A_{\text {proj, horiz }}}=\frac{F_{n}}{k_{\text {colon }}\left(W L-n W_{h} L_{h}\right)} \text {. }
$$

By combining Eqs. (A1)-(A9), friction force of a rectangular plate with holes can be calculated as

$$
\begin{aligned}
F_{\text {fric }}= & s_{\text {colon }}\left(W L-n W_{h} L_{h}\right) \\
& +\frac{F_{n}}{W L-n W_{h} L_{h}+2\left[(W+L)+n\left(W_{h}-L_{h}\right)\right]\left[F_{n} / k_{\text {colon }}\left(W L-n W_{h} L_{h}\right)\right]+4(1-n)\left\{\left[F_{n} / k_{\text {colon }}\left(W L-n W_{h} L_{h}\right)\right]\right\}^{2}} \\
& \times H_{\text {colon }} 2\left[\left(f_{\text {weight } W} W+f_{\text {weight } L} L\right)+n\left(f_{\text {weight } W} W_{h}+f_{\text {weight } L} L_{h}\right)\right] d_{\text {vert }} .
\end{aligned}
$$


For $n=0,(\mathrm{~A} 10)$ is reduced to (14), which calculates the friction force for a rectangular geometry with no holes.

\section{Circular geometries}

Consider a circular plate with radius $R$. In such a circular geometry, the resistant force should be split into two components, one parallel and one perpendicular to the tangent at each point of the circumference. In this case, the horizontal projected area can be calculated as

$$
A_{\text {proj,horiz }}=\pi R^{2} \text {, }
$$

whereas the vertical projected area as

$$
A_{\text {proj,vert }}=2 \pi R d_{\text {vert }} \int_{0}^{\pi / 2}\left(f_{\text {weight } W} \cos \theta+f_{\text {weight } L} \sin \theta\right) d \theta .
$$

The area correction is

$$
A_{\text {deform }}=\pi\left(R+d_{\text {horiz }}\right)^{2}-\pi R^{2},
$$

whereas

$$
d_{\text {horiz }}=\frac{F_{n}}{k_{\text {colon }} A_{\text {proj, horiz }}}=\frac{F_{n}}{k_{\text {colon }} \pi R^{2}} .
$$

By combining Eqs. (A1)-(A3), (A6), and (A11)-(A14), friction force of a circular plate can be calculated as

$$
\begin{aligned}
F_{\text {fric }}= & s_{\text {colon }} \pi R^{2}+\frac{F_{n}}{\left[R+\left(F_{n} / k_{\text {colon }} \pi R^{2}\right)\right]^{2}} H_{\text {colon }} 2 R d_{\text {vert }} \\
& \times \int_{0}^{\pi / 2}\left(f_{\text {weight } W} \cos \theta+f_{\text {weight } L} \sin \theta\right) d \theta .
\end{aligned}
$$

Circular geometries with one or more eccentric holes of the same size

Consider a circular plate with radius $R$. The plate includes $n$ holes of radius $R_{h}$. The horizontal projected area can in this case be calculated as

$$
A_{\text {proj,horiz }}=\pi\left(R^{2}-n R_{h}^{2}\right) .
$$

The vertical projected area is

$$
\begin{aligned}
A_{\text {proj,vert }}= & 2 \pi\left(R+n R_{h}\right) d_{\text {vert }} \\
& \times \int_{0}^{\pi / 2}\left(f_{\text {weight } W} \cos \theta+f_{\text {weight } L} \sin \theta\right) d \theta .
\end{aligned}
$$

The area correction around the plate is

$$
A_{\text {deform,o }}=\pi\left(R+d_{\text {horiz }}\right)^{2}-\pi R^{2},
$$

whereas the area correction inside the holes is

$$
A_{\text {deform }, i}=n \pi R_{h}^{2}-n \pi\left(R_{h}-d_{\text {horiz }}\right)^{2} .
$$

The horizontal distance $d_{\text {horiz }}$ is determined as

$$
d_{\text {horiz }}=\frac{F_{n}}{k_{\text {colon }} A_{\text {proj, horiz }}}=\frac{F_{n}}{k_{\text {colon }} \pi\left(R^{2}-n R_{h}^{2}\right)} .
$$

By combining Eqs. (A1)-(A3), (A6), and (A16)-(A20), friction force of a circular plate with holes can be calculated as

$$
\begin{aligned}
F_{\text {fric }}= & s_{\text {colon }} \pi\left(R^{2}-n R_{h}^{2}\right) \\
& +\frac{F_{n}}{\left(R^{2}-n R_{h}^{2}\right)+2\left(R+n R_{h}\right)\left[F_{n} / k_{\text {colon }} \pi\left(R^{2}-n R_{h}^{2}\right)\right]+(1-n)\left\{\left[F_{n} / k_{\text {colon }} \pi\left(R^{2}-n R_{h}^{2}\right)\right]\right\}^{2}} H_{\text {colon }} 2(R \\
& \left.+n R_{h}\right) d_{\text {vert }} \int_{0}^{\pi / 2}\left(f_{\text {weight } W} \cos \theta+f_{\text {weight } L} \sin \theta\right) d \theta .
\end{aligned}
$$

For $n=0,(\mathrm{~A} 21)$ is reduced to (A15), which calculates the friction force for a circular geometry with no holes.

\section{Triangular geometries}

Consider an equilateral triangular plate with side $T$. Along the two sides which create an angle different from 0 or 90 with the direction of the applied force, the resistant force is the result of two components, one parallel and one perpendicular to the tangent at each point. In this case, the horizontal projected area can be calculated as

$$
A_{\text {proj, horiz }}=\frac{\sqrt{3}}{4} T^{2}
$$

whereas the vertical projected area as

$$
A_{\text {proj,vert }}=T\left(1+f_{\text {weight } W} \cos \frac{\pi}{3}+f_{\text {weight } L} \sin \frac{\pi}{3}\right) .
$$

The area correction is

$$
A_{\text {deform }}=3 \sqrt{3}\left(\frac{\sqrt{3}}{6} T+d_{\text {horiz }}\right)^{2}-\frac{\sqrt{3}}{4} T^{2},
$$

whereas 


$$
d_{\text {horiz }}=\frac{F_{n}}{k_{\text {colon }} A_{\text {proj, horiz }}}=\frac{F_{n}}{k_{\text {colon }}(\sqrt{3} / 4) T^{2}} .
$$

By combining Eqs. (A1)-(A3), (A6), and (A22)-(A25), friction force of a triangular plate can be calculated as

$$
\begin{aligned}
F_{\text {fric }}= & S_{\text {colon }} \frac{\sqrt{3}}{4} T^{2}+\frac{F_{n}}{3\left[(1 / 2) T+\left(4 F_{n} / k_{\text {colon }} T^{2}\right)\right]^{2}} \\
& \times H_{\text {colon }} T\left(1+f_{\text {weight } W} \cos \frac{\pi}{3}+f_{\text {weight } L} \sin \frac{\pi}{3}\right) .
\end{aligned}
$$

${ }^{1}$ M. L. Anderson, T. M. Pasha, and J. A. Leighton, Am. J. Gastroenterol 95, 3418 (2000)

${ }^{2}$ S. M. Kavic and M. D. Basson, Am. J. Surg. 181, 319 (2001).

${ }^{3}$ P. Dario, M. C. Carrozza, and A. Pietrabissa, Comput. Aided Surg. 4, 1 (1999).

${ }^{4}$ L. Phee, D. Accoto, A. Menciassi, C. Stefanini, M. C. Carrozza, and P. Dario, IEEE Trans. Biomed. Eng. 49, 613 (2002).

${ }^{5}$ D. Dodou, P. Breedveld, and P. A. Wieringa, Minimally Invasive Ther. Allied Technol. 14, 188 (2005).

${ }^{6}$ D. Dodou, D. Girard, P. Breedveld, and P. A. Wieringa, Proceedings of International Conference on Advanced Robotics (IEEE, Seattle, WA, 2005), p. 352.
${ }^{7}$ D. Dodou, F. Bedaux, R. van Heffen, P. Breedveld, and P. A. Wieringa, J. Adhesion (in press).

${ }^{8}$ J. Comyn, Adhesion Science (Royal Society of Chemistry, UK, 1997), p. 114.

${ }^{9}$ G. S. Giare, D. J. Mulhollad, and R. F. Felton, Eng. Fract. Mech. 41, 487 (1992).

${ }^{10}$ D. Dodou, P. Breedveld, and P. A. Wieringa, Eur. J. Pharm. Biopharm. 60, 1 (2005).

${ }^{11}$ Carbopol, Nomenclature and Chemistry (Products information), BFGoodrich Company, USA: Noveon (2002).

${ }^{12}$ M. Muramatsu et al., Int. J. Pharm. 199, 77 (2000).

${ }^{13}$ C. Eouani, Ph. Piccerelle, P. Prinderre, E. Bourret, and J. Joachim, Eur. J. Pharm. Biopharm. 52, 45 (2001).

${ }^{14}$ N. C. Sanchez, P. L. Tenofsky, J. M. Dort, L. Y. Shen, S. D. Helmer, and R. S. Smith, Am. Surgeon 67, 243 (2001).

${ }^{15}$ R. A. Kozarek, D. S. Earnest, M. E. Silverstein, and R. G. Smith, Gastroenterology 78, 7 (1980).

${ }^{16}$ F. P. Bowden, A. J. W. Moore, and D. Tabor, J. Appl. Phys. 14, 80 (1943).

${ }^{17}$ C.-M. Lehr, J. A. Bouwstra, F. Spies, J. Onderwater, J. van het Noordeinde, C. Vermeij-Keers, C. J. van Munsteren, and H. E. Junginger, J. Controlled Release 18, 249 (1992).

${ }^{18}$ J. C. Barbenel and P. A. Payne, Bioengineering \& the Skin Newsletter 3, 8 (1981).

${ }^{19}$ W. J. O'Brien, Biomaterials Properties Database, University of Michigan (www.lib.umich.edu/dentlib/Dental_tables/ShoreAhard.html)

${ }^{20}$ H. Hägerström and K. Edsman, J. Pharm. Pharmacol. 53, 1589 (2001).

${ }^{21}$ J. D. Smart, Adv. Drug Delivery Rev. 57, 1556 (2005).

${ }^{22}$ D. Gersappe and M. O. Robbins, Europhys. Lett. 48, 150 (1999). 